\title{
New Bounds for the Principal Dirichlet Eigenvalue of Planar Regions
}

\author{
Pedro Antunes and Pedro Freitas
}

\section{CONTENTS}

1. Introduction

2. The Numerical Method

3. Results for $\lambda_{1}$ on Polygons

4. One-Term Bounds

5. Two-Term Bounds

6. Discussion

Acknowledgments

References

2000 AMS Subject Classification: Primary 35P15; Secondary 58G25

Keywords: Dirichlet eigenvalues, Pólya-Szegő conjectures
We present a numerical study for the first Dirichlet eigenvalue of certain classes of planar regions. Based on this, we propose new types of bounds and establish several conjectures regarding the dependence of this eigenvalue on the perimeter and the area.

\section{INTRODUCTION}

The well-known Faber-Krahn inequality states that of all sufficiently regular bounded domains with the same volume, the ball has the smallest first Dirichlet eigenvalue. In the case of a planar domain $\Omega$ with area $A$, this can be stated as

$$
\lambda_{1}(\Omega) \geq \frac{\pi j_{0,1}^{2}}{A},
$$

where $j_{0,1}$ is the first positive zero of the Bessel function $J_{0}$, and equality holds for the disk.

If we now restrict the class of domains under consideration, it is possible to improve the above result. This can be done in several different ways, of which we shall now discuss some examples.

One possibility is to consider the class of $n$-polygons, for which Pólya and Szegó proposed the following in [Pólya and Szegó 51].

Conjecture 1.1. Of all n-polygons with the same area, the regular n-polygon has the smallest first Dirichlet eigenvalue.

Using Steiner symmetrization, Pólya and Szegő proved the conjecture for the case of triangles and quadrilaterals in [Pólya and Szegó 51], and as far as we are aware, no progress whatsoever has been made on this problem over the last forty years.

If we denote by $P_{n}^{\text {reg }}$ the regular $n$-polygon of unit area, Pólya and Szegö's conjecture may be stated, in the same fashion as (1-1), as the following inequality for the 
first Dirichlet eigenvalue of an $n$-polygon of area $A$ :

$$
\lambda_{1}\left(P_{n}\right) \geq \frac{1}{A} \lambda_{1}\left(P_{n}^{\mathrm{reg}}\right),
$$

with equality if and only if $P_{n}$ is the regular $n$-polygon. For the case of triangles and quadrilaterals this yields

$$
\lambda_{1}\left(P_{3}\right) \geq \frac{4 \sqrt{3} \pi^{2}}{3 A} \quad \text { and } \quad \lambda_{1}\left(P_{4}\right) \geq \frac{2 \pi^{2}}{A} .
$$

These bounds are isoperimetric inequalities of the same type as the Faber-Krahn bound, and indeed, if the Pólya-Szegő conjecture holds, the Faber-Krahn inequality can be seen as the limit of (1-2) as $n$ goes to infinity. Their limitation is that since the estimate is based only on the area of the domain under consideration, they do not take into account any measure of the deviation from the optimizer. In order to do this, one normally needs to restrict more the class of domains for which the inequality will be applicable. If we consider, for instance, the class of convex planar domains $\mathcal{K}$, we have that for all $K \in \mathcal{K}$,

$$
\frac{\pi^{2} L^{2}}{16 A^{2}} \leq \lambda_{1}(K) \leq \frac{\pi^{2} L^{2}}{4 A^{2}},
$$

where $A$ and $L$ denote the area and boundary length of $K$, respectively. The left-hand inequality is due to Makai [Makai 62], while the right-hand inequality was proven by Pólya in [Pólya 60], and as was pointed out in [Osserman 77], it actually holds for arbitrary doubly connected domains; see the examples regarding this issue given in the last section. Note also that as has been pointed out in [Freitas 06], it is possible to improve on the lower bound in (1-3). This is done using Protter's bound [Protter 81]

$$
\lambda_{1}(K) \geq \frac{\pi^{2}}{4}\left[\frac{1}{\rho^{2}}+\frac{1}{d^{2}}\right],
$$

where $\rho$ and $d$ denote the in-radius and the diameter of $K$, respectively. Following Makai's argument one is then led to

$$
\lambda_{1}(K) \geq \frac{\pi^{2}}{4}\left[\frac{L^{2}}{4 A^{2}}+\frac{1}{d^{2}}\right] .
$$

All this suggests that one might try looking for improved bounds of the same form as (1-3) under the restriction that $K$ is now an $n$-polygon, which, in the case of lower bounds, will be assumed to be convex. However, since the right- and left-hand-side inequalities in (1-3) are attained in the limiting case of a long thin rectangle and a narrow circular sector, respectively, this will not be possible in general, and one has thus to look for bounds that include the quantities $L$ and $A$ in a more involved way; see, however, Conjecture 5.1 and [Freitas 06].

Since, as illustrated by the Pólya-Szegö conjecture, these problems are notoriously difficult, we set out to obtain numerical data that would allow us to uncover any underlying structure of the dependence of the first eigenvalue on $L$ and $A$. For instance, since there is clearly a relation between geometric and spectral isoperimetric inequalities, an obvious question is whether for fixed area there are any conditions under which the first eigenvalue is a monotone function of the length of the boundary. That this cannot be true for general convex domains may be seen by considering an isosceles right triangle $T$ with area $\frac{1}{2}$, and a rectangle $R$ of sides $\frac{5}{4}$ and $\frac{2}{5}$, for which we have

$$
L(R)=\frac{33}{10}<2+\sqrt{2}=L(T),
$$

while

$$
\lambda_{1}(R)=\frac{689}{100} \pi^{2}>5 \pi^{2}=\lambda_{1}(T) .
$$

We will see that even when we consider this issue in more restricted classes such as triangles, there exists no direct relation between the monotonicity of the perimeter and that of the first Dirichlet eigenvalue.

The other point we address is the existence of more precise bounds for the first eigenvalue, and what quantities should be involved here. It is our belief that at the present stage, it would be useful to obtain some insight into how the different quantities should be related, and that this is best done numerically. Indeed, the data gathered suggest some new and, we hope, fruitful research directions in this area. As an example, we single out the new type of bounds that take on the form of the functions $F_{n}$ defined below, and the fact that, depending on the choice of parameters, one may obtain both upper and lower bounds.

The main purpose of this paper is thus to investigate numerically new types of bounds for the first Dirichlet eigenvalue of polygons based on the considerations made above. We will explore two different variations on this. The first is to look for bounds that add a term that will take into account the length of the boundary. A natural way of doing this is to consider expressions of the form

$$
\frac{c_{1}}{A}+c_{2} \frac{L^{2}-\kappa_{n} A}{A^{2}},
$$

where $c_{1}$ and $c_{2}$ are constants to be determined, and

$$
\kappa_{n}=4 n \tan \frac{\pi}{n}
$$

is the corresponding isoperimetric constant, that is, $\kappa_{n}$ is such that for any $n$-polygon one has $L^{2}-\kappa_{n} A \geq 0$, 
with equality if and only if the polygon is regular. In this way, one is including a correction due to what might be called the isoperimetric defect of the polygon. Such an idea is not new and was already used in [Payne and Weinberger 61$]$, where it was shown that for a bounded simply connected domain $\Omega$ in $\mathbb{R}^{2}$, one has

$$
\lambda_{1}(\Omega) \leq \frac{\pi j_{0,1}^{2}}{A}\left[1+\left(\frac{1}{J_{1}^{2}\left(j_{0,1}\right)}-1\right)\left(\frac{L^{2}}{4 \pi A}-1\right)\right]
$$

Here $J_{1}$ is the Bessel function of the first kind of order 1 . What is new in our approach is that we study the possibility of extending bounds of the above type to polygons with $n$ sides, and not only as upper bounds, but also as lower bounds. Moreover, the limiting case we obtain for the upper bounds as we let the number of sides go to infinity suggests an upper bound for simply connected domains that improves on (1-5) and is in agreement with the numerical studies that were carried out; see Conjecture 6.1.

Rewriting the terms in (1-4) in a more convenient way, we shall thus look for bounds of the form

$$
F_{n}(L, A, \alpha, \beta)=\pi^{2} \frac{L^{2}-\alpha A}{\beta A^{2}},
$$

where $\alpha$ and $\beta$ are constants to be determined for each $n$. We point out that if one writes down an explicit expression for the first eigenvalue of a rectangle in terms of its area and perimeter, one is led to the expression

$$
\lambda_{1}(R)=F_{4}(L, A, 8,4)=\pi^{2} \frac{L^{2}-8 A}{4 A^{2}} .
$$

An interesting feature of these expressions is that, as was mentioned above, depending on the values of the parameters $\alpha$ and $\beta$, the numerical data gathered indicate that there exist both lower and upper bounds that are of this form. In the case of the lower bound, we shall present a conjecture that if true, implies Pólya and Szegó's conjecture in the convex case; see Conjecture 5.2.

Another set of bounds that we shall explore in this paper is related to the bounds given by (1-3). Namely, we shall now consider the way in which the exponents in those expressions are allowed to vary. To this end, we shall consider functions of the form

$$
G_{n}(L, A, \alpha, q)=\alpha \frac{L^{2(q-1)}}{A^{q}} .
$$

As with the case of the bounds given by $F_{n}$, this will allow us to obtain both upper and lower bounds for the first eigenvalue of $n$-polygons.
The organization of the paper is as follows. We begin by describing the numerical method used. In Section 3 we investigate the behavior of the first eigenvalue of convex polygons of fixed area and show that even in the case of triangles, $\lambda_{1}$ does not behave monotonically with respect to the perimeter.

Then, in Sections 4 and 5, we present numerical data obtained for one- and two-term bounds, of the form $G_{n}$ and $F_{n}$, respectively, and, based on the results obtained, present a series of conjectures. In the last section we discuss the results obtained.

\section{THE NUMERICAL METHOD}

Let $\Omega \subset \mathbb{R}^{2}$ be a bounded domain. We will consider the two-dimensional Dirichlet eigenvalue problem for the Laplace operator. This is equivalent to determining the resonance frequencies $\kappa$ that satisfy the Dirichlet problem for the Helmholtz equation

$$
\begin{aligned}
\Delta u+\kappa^{2} u=0 & \text { in } \Omega, \\
u=0 & \text { on } \partial \Omega,
\end{aligned}
$$

for a nonnull function $u$.

We will describe the approach to this problem with a mesh-free method, the method of fundamental solutions. For details, see [Alves and Antunes 05].

A fundamental solution $\Phi_{\kappa}$ of the Helmholtz equation satisfies

$$
\left(\Delta+\kappa^{2}\right) \Phi_{\kappa}=-\delta,
$$

where $\delta$ is the Dirac delta distribution. In the twodimensional case, we take

$$
\Phi_{\kappa}(x)=\frac{i}{4} H_{0}^{(1)}(\kappa|x|)
$$

where $H_{0}^{(1)}$ is the first Hänkel function.

Let $\hat{\Gamma}$ be, for instance, the boundary of a bounded open set $\hat{\Omega} \supset \bar{\Omega}$, considering $\hat{\Gamma}$ as surrounding $\partial \Omega$. A density result obtained in 2000 by Alves [Alves 00] states that if $\kappa$ is not an eigenfrequency, then

$$
\overline{\operatorname{span}\left\{\left.\Phi_{\kappa}(x-y)\right|_{x \in \partial \Omega}: y \in \hat{\Gamma}\right\}}=L^{2}(\partial \Omega) .
$$

This result allows us to approximate an $L^{2}$ function defined on $\partial \Omega$, with complex values, using a sequence of functions

$$
u_{m}(x)=\sum_{j=1}^{m} \alpha_{m, j} \Phi_{\kappa}\left(x-y_{m, j}\right)
$$


that converges to $\left.u\right|_{\Gamma}$ in $L^{2}(\partial \Omega)$. Every function of the form (2-2) satisfies the Helmholtz equation in the domain $\Omega$ because $\bigcup_{j=1}^{m}\left\{y_{m, j}\right\} \subset \bar{\Omega}^{C}$.

We will define $m$ collocation points $x_{i} \in \partial \Omega$ and $m$ source points $y_{m, j} \in \hat{\Gamma}$ to obtain the system

$$
\sum_{j=1}^{m} \alpha_{m, j} \Phi_{\kappa}\left(x_{i}-y_{m, j}\right)=0, \quad x_{i} \in \partial \Omega .
$$

This allows us to approximate the boundary condition of problem $(2-1)$. Therefore the numerical algorithm to calculate the eigenfrequencies is to find the values $\kappa$ for which the $m \times m$ matrix

$$
A(\kappa)=\left[\Phi_{\kappa}\left(x_{i}-y_{j}\right)\right]_{m \times m}
$$

has a null determinant. We consider a particular choice of the points $x_{1}, \ldots, x_{m} \in \partial \Omega$ and $y_{1}, \ldots, y_{m} \in \hat{\Gamma}$ described in [Alves and Antunes 05].

The components of the matrix $A(\kappa)$ are complex numbers, so the determinant is also a complex number. We consider the real function $g(\kappa)=|\operatorname{det}[A(\kappa)]|$. If $\kappa$ is an eigenfrequency, $\kappa$ is a minimizer where $g(\kappa)=0$. To approximate the eigenfrequencies we calculate the points where the local minima are attained using an algorithm based on the golden-ratio search method.

Once we have an eigenfrequency determined, we may obtain the eigenvalue just by calculating $\lambda=\kappa^{2}$.

To obtain an eigenfunction associated with the eigenfrequencies $\kappa_{1}, \kappa_{2}, \ldots$, we use a collocation method on $m+1$ points, with $x_{1}, \ldots, x_{m}$ on $\partial \Omega$ and a point $x_{m+1} \in$ $\Omega$. Then, the approximated eigenfunction is given by

$$
\tilde{u}(x)=\sum_{k=1}^{m+1} \alpha_{k} \Phi_{k_{i}}\left(x-y_{k}\right),
$$

and to exclude the solution $\tilde{u}(x) \equiv 0$, the coefficients $\alpha_{k}$ are determined by the resolution of the system

$$
\begin{aligned}
\tilde{u}\left(x_{i}\right) & =0, \quad i=1, \ldots, m, \\
\tilde{u}\left(x_{m+1}\right) & =1 .
\end{aligned}
$$

\section{RESULTS FOR $\lambda_{1}$ ON POLYGONS}

As a first approach we considered a series of polygons with the number of sides varying between three and eight, and plotted the first Dirichlet eigenvalue as a function of the perimeter; see Figure 1.

The results obtained show that in general, $\lambda_{1}$ is not necessarily increasing with the length of the boundary. An example of this can be seen by considering the two
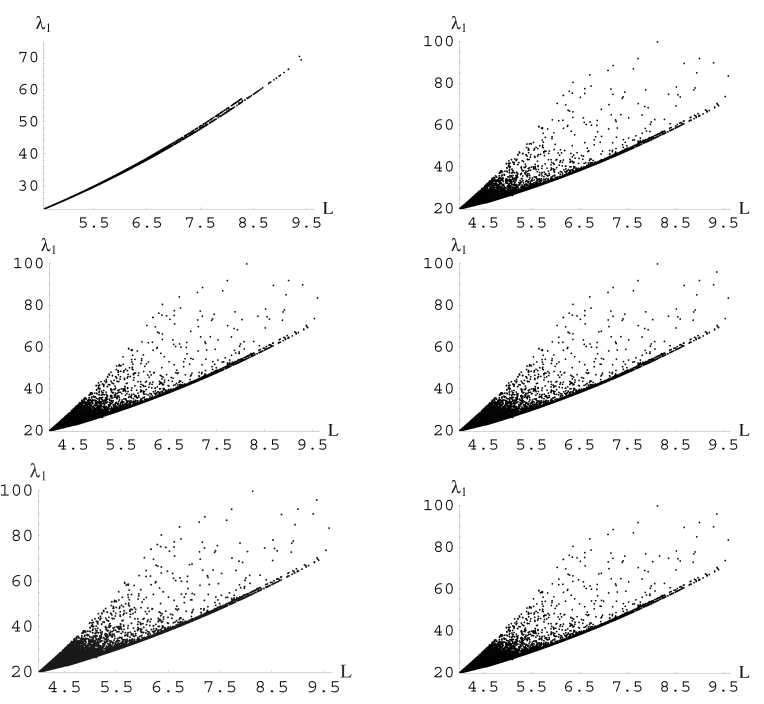

FIGURE 1. Plots of $\lambda_{1}(L)$ for convex $n$-polygons of unit area with $n=3,4,5,6,7,8$.

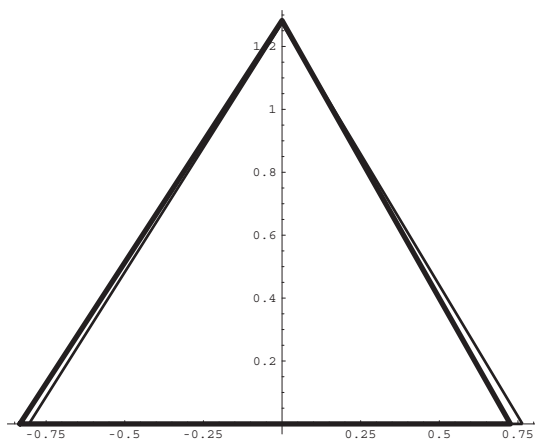

FIGURE 2. Plot of the triangles $T_{1}$ (thin line) and $T_{2}$ (bold line).

triangles $T_{1}$ and $T_{2}$ with unit area, which are shown in Figure 2.

We believe that all the digits presented in Table 1 for the values of $\lambda_{1}$ are correct. The triangle $T_{2}$ has a smaller perimeter than $T_{1}$, and we shall prove numerically that $T_{2}$ has a larger value of $\lambda_{1}$. In order to do this, we will use a result from [Fox et al. 67] that was simplified and extended in [Moler and Payne 68].

\begin{tabular}{|c|c|c|}
\hline & $T_{1}$ & $T_{2}$ \\
\hline \multirow{3}{*}{ angles (degrees) } & 59.05014828 & 60.49209561 \\
& 57.74089539 & 56.97940476 \\
& 63.20895633 & 62.52849962 \\
\hline$L$ & 4.56271413 & 4.56271232 \\
\hline$\lambda_{1}$ & 22.81833704 & 22.81835620 \\
\hline
\end{tabular}

TABLE 1. Perimeter and $\lambda_{1}$ for the triangles $T_{1}$ and $T_{2}$. 
Let $\lambda$ and $u$ be an eigenvalue and eigenfunction satisfying the Dirichlet problem

$$
\begin{aligned}
-\Delta u=\lambda u & & \text { in } \Omega, \\
u=0 & & \text { on } \partial \Omega .
\end{aligned}
$$

In general, the numerical method that is used allows us to obtain $\tilde{\lambda}$ and $\tilde{u}$, an approximate eigenvalue and eigenfunction satisfying not problem (3-1), but

$$
\begin{aligned}
-\Delta \tilde{u} & =\tilde{\lambda} \tilde{u} & & \text { in } \Omega, \\
\tilde{u} & =\epsilon(x) & & \text { on } \partial \Omega,
\end{aligned}
$$

where $\epsilon(x)$ is a function that is small on $\partial \Omega$.

Theorem 3.1. If $\tilde{\lambda}$ and $\tilde{u}$ satisfy problem (3-2), there exists an (exact) eigenvalue $\lambda_{k}$ of problem (3-1) such that

$$
\frac{\left|\lambda_{k}-\tilde{\lambda}\right|}{\lambda_{k}} \leq \theta,
$$

where

$$
\theta=\frac{\sqrt{A} \sup _{x \in \partial \Omega}|\epsilon(x)|}{\|\tilde{u}\|_{L^{2}(\Omega)}} .
$$

If in addition, $\|\tilde{u}\|_{L^{2}(\Omega)}=1$ and $u_{k}$ is the normalized orthogonal projection of $u$ onto the eigenspace of $\lambda_{k}$, then

$$
\left\|u_{k}-\tilde{u}\right\|_{L^{2}(\Omega)} \leq \frac{\theta}{\rho_{k}}\left(1+\frac{\theta^{2}}{\rho_{k}^{2}}\right)^{1 / 2}
$$

where

$$
\rho_{k}:=\min _{\lambda_{n} \neq \lambda_{k}} \frac{\left|\lambda_{n}-\tilde{\lambda}\right|}{\lambda_{n}} .
$$

Using inequality (3-3) we obtain

$$
\left(\frac{1}{1+\theta}\right) \tilde{\lambda} \leq \lambda_{k} \leq\left(\frac{1}{1-\theta}\right) \tilde{\lambda}
$$

yielding the results for the triangles $T_{1}$ and $T_{2}$ shown in Table 2, where the eigenfunction was obtained with the procedure described in Section 2 and the integral was calculated numerically. To obtain an approximation for $\sup _{x \in \partial \Omega}|\epsilon(x)|$ we calculate $\max _{i}\left|\tilde{u}\left(z_{i}\right)\right|$, where the $z_{i}$ are 1001 points on $\partial \Omega$.

\begin{tabular}{|c|c|c|}
\hline & $T_{1}$ & $T_{2}$ \\
\hline $\sup _{x \in \partial \Omega} \epsilon(x)$ & $4.077068 \times 10^{-8}$ & $8.0378213 \times 10^{-8}$ \\
\hline$\|\tilde{u}\|_{L^{2}(\Omega)}$ & 0.637602 & 0.620147 \\
\hline
\end{tabular}

TABLE 2. Results for the approximations of the eigenfunctions of $T_{1}$ and $T_{2}$.

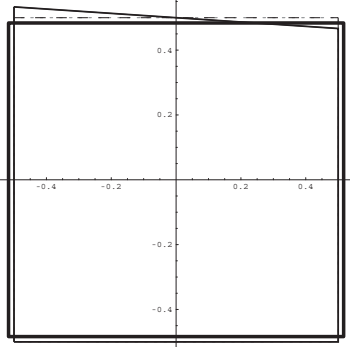

FIGURE 3. Plot of the boundary of the polygons $Q_{1}$ (bold line), $Q_{2}$ (thin line), and the square (dashed line).

\begin{tabular}{|c|c|c|c|}
\hline & $Q_{1}$ & $Q_{2}$ & $P_{4}^{\text {reg }}$ \\
\hline $\mathrm{L}$ & 4.00219305 & 4.00221975 & 4 \\
\hline$\lambda_{1}$ & 19.782509 & 19.752103 & 19.739208 \\
\hline
\end{tabular}

TABLE 3. Perimeter and $\lambda_{1}$ for the quadrilaterals $Q_{1}$, $Q_{2}$ and $P_{4}^{\text {reg }}$.

Applying the bounds (3-7), we obtain

$$
22.81835437<\lambda_{1}\left(T_{2}\right)<22.81835803
$$

and

$$
22.81833558<\lambda_{1}\left(T_{1}\right)<22.81833850,
$$

so

$$
\lambda_{1}\left(T_{1}\right)<\lambda_{1}\left(T_{2}\right) .
$$

The lack of general monotonicity on the perimeter for the case of convex quadrilaterals is easier to observe. Let $Q_{1}$ and $Q_{2}$ be the quadrilaterals with unit area shown in Figure 3. Here $Q_{1}$ is a rectangle with side lengths $3101 / 3000$ and $3000 / 3101$, and $Q_{2}$ has vertices

$$
\left(-\frac{1}{2},-\frac{1}{2}\right),\left(\frac{1}{2},-\frac{1}{2}\right),\left(\frac{1}{2}, \frac{7}{15}\right),\left(-\frac{1}{2}, \frac{8}{15}\right) \text {. }
$$

We obtain the results shown in Table 3 . All the digits presented for the values of $P_{4}^{\text {reg }}$ and $Q_{1}$ are correct, since we have an explicit expression for the eigenvalues

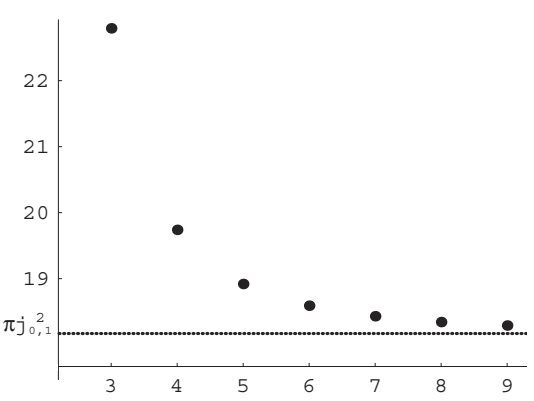

FIGURE 4. $\lambda_{1}(n)$ for the polygons $P_{n}^{\text {reg }}$ with unit area, $3 \leq n \leq 9$. 


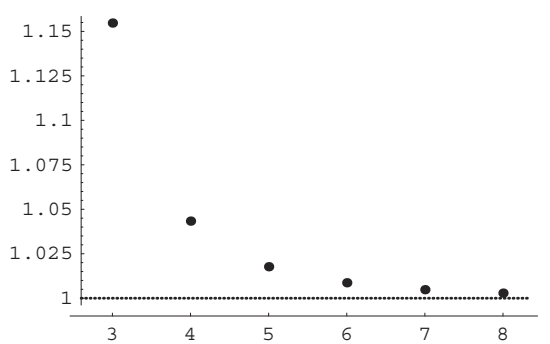

FIGURE 5. $q_{n}$ for the polygons $P_{n}^{\text {reg }}$ with unit area, $3 \leq n \leq 8$.

of rectangles. We believe that all the digits presented for $Q_{2}$ are correct.

In Figure 4 we represent the first eigenvalue $\lambda_{1}$ for the first seven regular polygons $P_{n}^{\text {reg }}$ with unit area. The dotted curve denotes the limiting case of the first eigenvalue of the disk. In Figure 5 we represent the quotients

$$
q_{n}:=\frac{\lambda_{1}\left(P_{n}^{\text {reg }}\right)}{\lambda_{1}\left(P_{n+1}^{\text {reg }}\right)} .
$$

These results suggest the following conjecture.

\section{Conjecture 3.2. It is conjectured that}

$$
\lambda_{1}\left(P_{3}^{\text {reg }}\right)>\lambda_{1}\left(P_{4}^{\text {reg }}\right)>\cdots>\lambda_{1}(B),
$$

where $B$ is the disk. Furthermore, we have $q_{3}>q_{4}>$ $q_{5}>\cdots$.

Remark 3.3. Note that Conjecture 1.1 trivially implies the first part of Conjecture 3.2.

\section{ONE-TERM BOUNDS}

In the introduction, we recalled some known estimates involving the terms $1 / A$ and $L^{2} / A^{2}$. These are particular instances of more general terms of the type

$$
\frac{L^{2(q-1)}}{A^{q}}, \quad q \geq 1 .
$$

We shall now consider bounds based on

$$
G_{n}(L, A, \alpha, q)=\alpha \frac{L^{2(q-1)}}{A^{q}}, \quad q \geq 1 .
$$

Consider first the case of triangles, for which we shall determine the value of $\alpha$ as a function of the exponent $q$, so that equality holds for the case of the equilateral triangle. This yields

$$
\alpha=3^{(1-3 q / 2)} 4^{(2-q)} \pi^{2} .
$$
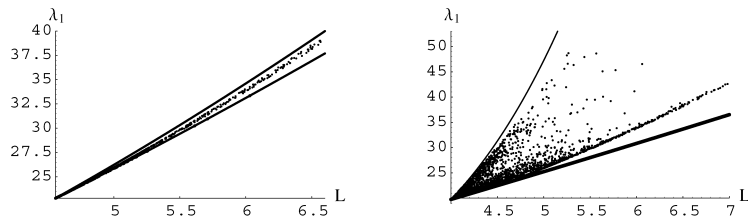

FIGURE 6. Plots of $\lambda_{1}(L)$ and the corresponding lower and upper bounds for triangles and quadrilaterals of unit area.

Then we choose the values of $q$ giving lower and upper bounds for all the triangles considered. Proceeding in this way, we are led to the following estimates:

$$
\begin{aligned}
& \lambda_{1}(L, A) \leq G_{3}\left(L, A, \alpha_{0}, q\right), \quad q \geq q_{0}, \\
& \lambda_{1}(L, A) \geq G_{3}\left(L, A, \alpha_{0}, q\right), \quad q \leq q_{1},
\end{aligned}
$$

with $q_{0} \approx 1.76$ and $q_{1} \approx 1.68$.

In Figure 6 we show the plots of $\lambda_{1}(L)$ together with the corresponding lower and upper bounds for the case of triangles of unit area.

For the case of quadrilaterals, we observed numerically that the best lower bound gives equality in the cases of both the square and the equilateral triangle. We obtain $\alpha=2^{(5-4 q)} \pi^{2}, q_{0} \approx 2.96$, and $q_{1} \approx 1.54$. The corresponding results are shown in Figure 6.

For the remaining $n$-polygons we followed the same procedure to determine the two constants $q_{0}$ and $q_{1}$ giving lower and upper bounds, respectively. We observed numerically that choosing the value of the parameters $\alpha$ and $q$ such that equality holds in the case of regular polygons $P_{n}^{\text {reg }}$ and $P_{n-1}^{\text {reg }}$ yields lower bounds. In this case the system to obtain the parameters is nonlinear, but it can easily be solved, for instance, with Newton's method for systems.

\section{TWO-TERM BOUNDS}

\subsection{Triangles}

We shall now present our results for bounds of the type (1-4). As before, we begin with the case of triangles, for which we look for bounds of the type

$$
\begin{aligned}
E_{3}(L, A, \theta) & :=F_{3}\left(L, A, 12 \sqrt{3}-\frac{4 \pi^{2}}{\sqrt{3} \theta}, \frac{\pi^{2}}{\theta}\right) \\
& =\frac{4 \pi^{2}}{\sqrt{3} A}+\theta \frac{L^{2}-12 \sqrt{3} A}{A^{2}} .
\end{aligned}
$$

From the values obtained for the triangles considered, we conjecture that

$$
\begin{array}{ll}
\lambda_{1}(L, A) \leq E_{3}(L, A, \theta), & \theta \geq \theta_{0}, \\
\lambda_{1}(L, A) \geq E_{3}(L, A, \theta), & \theta \leq \theta_{1},
\end{array}
$$


with $\theta_{0} \approx 0.77$ and $\theta_{1} \approx 0.67$ (see Figure 7 ). In particular, we conjecture that for triangles there is a simple upper bound that is better than the more general bound of Pólya's given in (1-3):

Conjecture 5.1. For any triangle $T$ we have

$$
\lambda_{1}(T) \leq \frac{\pi^{2} L^{2}}{9 A^{2}} .
$$

In this respect, we remark that for a triangle $T$ with sides of lengths $\ell_{1} \leq \ell_{2} \leq \ell_{3}$, it has recently been proven in [Freitas 06] that

$$
\pi^{2}\left(\frac{4}{\ell_{3}^{2}}+\frac{\ell_{3}^{2}}{4 A^{2}}\right) \leq \lambda_{1}(T) \leq \frac{\pi^{2}}{3 A^{2}}\left(\ell_{1}^{2}+\ell_{2}^{2}+\ell_{3}^{2}\right) .
$$

In the first plot of Figure 7 we present the values of $\lambda_{1}(L)$ for the unit-area triangles considered. We plot the lower and upper bounds that we obtained (bounds (5-2)); we also show (with a dashed line) the bound in Conjecture 5.1.

\subsection{Quadrilaterals}

A similar analysis may be carried out for quadrilaterals, and with the values obtained, we conjecture that we now have

$$
\pi^{2} \frac{(2 \sqrt{3}-3) L^{2}+4 \sqrt{3} A}{6(3 \sqrt{3}-4) A^{2}} \leq \lambda_{1}(Q) \leq \pi^{2} \frac{L^{2}-8 A}{4 A^{2}} .
$$

The numerical evidence gathered seems to indicate that these will be the best estimates involving the terms $\frac{1}{A}$ and $\frac{L^{2}}{A^{2}}$. Equality for the lower bound is again attained in the case of both the square and the equilateral triangle. Equality holds in the upper bound for rectangles. Note that now, in contrast to the case of triangles, it is not possible to have an upper bound of the form $c(L / A)^{2}$ that would improve on that of Pólya in inequality (1-3), since this latter bound is attained asymptotically for long thin rectangles; we can also see that the value of $\theta$ for which we have only the $(L / A)^{2}$ term is no longer within the range giving upper bounds. Based on the upper bound for triangles in (5-3), we will, however, be able to suggest another class of bounds of that type for general polygons; see Section 5.3 below. In the case of a quadrilateral of side lengths $\ell_{i}, i=1, \ldots, 4$, this reduces to

$$
\lambda_{1}(Q) \leq \frac{\pi^{2}}{2 A^{2}} \sum_{i=1}^{4} \ell_{i}^{2}
$$

which is known to hold in the case of parallelograms [Hersch 66].
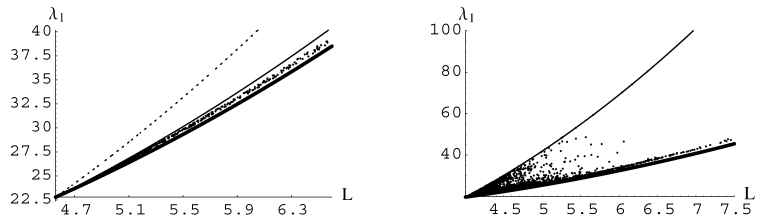

FIGURE 7. Plots of $\lambda_{1}(L)$ and the corresponding bounds for triangles and quadrilaterals.

The results for quadrilaterals (bounds (5-4)) are shown in the second plot in Figure 7.

5.2.1 Rhombic Membranes. The special case of the rhombus received considerable attention in the $1960 \mathrm{~s}$, not just theoretically but also from a numerical perspective [Hooker and Protter 60/61, Moler 69, Stadter 66]. The reasons for this are twofold. One the one hand, and as was stated in [Hooker and Protter 60/61], "it is a remarkable fact that the exact value is unknown in analytic form except in the case of a square (and the degenerate case of a slit or a strip)." On the other hand, the rhombus turns up naturally in the process of applying Steiner symmetrization to quadrilaterals.

In this section we compare our results for $\lambda_{1} / \pi^{2}$ to those given in [Moler 69, Stadter 66, Kuttler and Sigillito 85] for skew angles $\phi$, as shown in Figure 8.

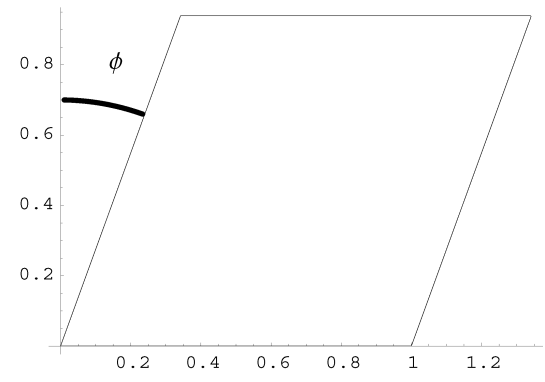

FIGURE 8. Rhombic domain.

Our bounds are obtained using Theorem 3.1, and so their accuracy depends on the approximation of the eigenfunction on the boundary of the domain. We are not able to obtain bounds better than Stadter's or KuttlerSigillito's bounds for large $\phi$, since the approximations for the eigenfunctions are too large on the boundary. However, for small $\phi$ our results are better than those of Stadter, Moler, and Kuttler-Sigillito. See Table 4. 


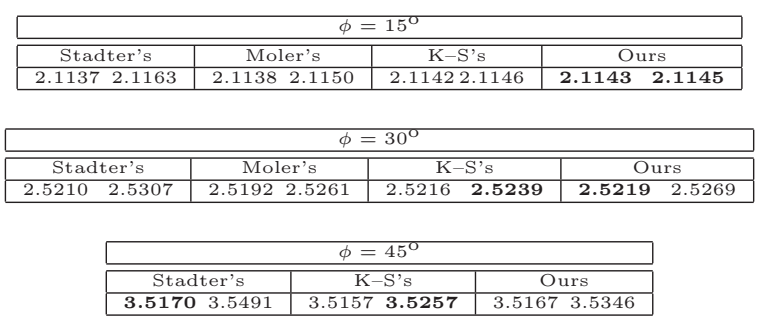

TABLE 4. Comparison of Stadter's, Moler's, KuttlerSigillito's (K-S), and our bounds for $\lambda_{1} / \pi^{2}$ on rhombical domains.

\subsection{General Polygons}

For other $n$-polygons the situation is similar, and we looked for bounds of the type

$$
\begin{aligned}
E_{n}(L, A, \theta) & :=F_{n}\left(L, A, \kappa_{n}-\frac{\lambda_{1}\left(P_{n}^{\mathrm{reg}}\right)}{\theta}, \frac{\pi^{2}}{\theta}\right) \\
& =\frac{\lambda_{1}\left(P_{n}^{\mathrm{reg}}\right)}{A}+\theta \frac{L^{2}-\kappa_{n} A}{A^{2}} .
\end{aligned}
$$

For each value of $n$, we obtained

$$
\begin{aligned}
& \lambda_{1}(L, A) \leq E_{n}(L, A, \theta), \theta \geq \theta_{0}, \\
& \lambda_{1}(L, A) \geq E_{n}(L, A, \theta), \theta \leq \theta_{1},
\end{aligned}
$$

for certain positive constants $\theta_{0}$ and $\theta_{1}$. In the case of pentagons we get $\theta_{0} \approx 2.467401$ and $\theta_{1} \approx 0.558268$. Note that the constant $\theta_{1}$ is chosen such that equality is attained not only for $P_{n}^{\text {reg }}$ but also in the case of the regular $(n-1)$-polygon. Based on this, we conjecture that for $n$-polygons it will be possible to obtain a bound of the form

$$
\lambda_{1} \geq \alpha \frac{1}{A}+\beta \frac{L^{2}}{A^{2}},
$$

where $\alpha$ and $\beta$ are such that we have equality in the cases of the regular polygons $P_{n}^{\text {reg }}$ and $P_{n-1}^{\text {reg }}$. More precisely, we have the following conjecture.

Conjecture 5.2. For convex n-polygons $P_{n}$ we have

$$
\begin{aligned}
\lambda_{1}\left(P_{n}\right) \geq & \frac{\kappa_{n-1} \lambda_{1}\left(P_{n}^{\mathrm{reg}}\right)-\kappa_{n} \lambda_{1}\left(P_{n-1}^{\mathrm{reg}}\right)}{\left(\kappa_{n-1}-\kappa_{n}\right) A} \\
& +\frac{\left[\lambda_{1}\left(P_{n-1}^{\mathrm{reg}}\right)-\lambda_{1}\left(P_{n}^{\mathrm{reg}}\right)\right] L^{2}}{\left(\kappa_{n-1}-\kappa_{n}\right) A^{2}} \\
= & \frac{\lambda_{1}\left(P_{n}^{\mathrm{reg}}\right)}{A} \\
& +\frac{\lambda_{1}\left(P_{n-1}^{\mathrm{reg}}\right)-\lambda_{1}\left(P_{n}^{\mathrm{reg}}\right)}{\kappa_{n-1}-\kappa_{n}}\left(\frac{L^{2}-\kappa_{n} A}{A^{2}}\right) .
\end{aligned}
$$

Remark 5.3. Note that Conjecture 5.2 implies Conjecture 1.1 and the first part of Conjecture 3.2 in the convex case.
We remark that we do not expect to obtain a new type of bound in the limiting case as $n$ goes to infinity. In fact, we conjecture that we have

$$
\lim _{n \rightarrow \infty} \frac{\lambda_{1}\left(P_{n-1}^{\mathrm{reg}}\right)-\lambda_{1}\left(P_{n}^{\mathrm{reg}}\right)}{\kappa_{n-1}-\kappa_{n}}=0,
$$

thus recovering the Faber-Krahn inequality.

In the case of upper bounds, and although it is possible to obtain the values of $\theta_{1}$ numerically, an explicit conjecture is not obvious, since it is not clear for which case other than the regular polygon one should force equality to hold. One possibility is to take into account the bound (5-4) for quadrilaterals, and in the case of general polygons, look for bounds that would give equality not only for the regular $n$-polygon but also for asymptotically long thin rectangles. Proceeding in this way, we are led to a bound of the form given by $(5-5)$ with $\theta=\pi^{2} / 4$.

Conjecture 5.4. For n-polygons $P_{n}$ we have

$$
\lambda_{1}\left(P_{n}\right) \leq \frac{\lambda_{1}\left(P_{n}^{\mathrm{reg}}\right)}{A}+\frac{\pi^{2}}{4} \frac{L^{2}-\kappa_{n} A}{A^{2}} .
$$

An alternative type of upper bound was already mentioned in the section on quadrilaterals, and was inspired by the upper bound for triangles obtained in [Freitas 06]. More precisely, based on this and on the numerical data available, we present the following conjecture.

Conjecture 5.5. For n-polygons $P_{n}$ with side lengths $\ell_{i}$, $i=1, \ldots, n$, we have

$$
\lambda_{1}\left(P_{n}\right) \leq\left(\frac{n}{\kappa_{n} A^{2}} \sum_{i=1}^{n} \ell_{i}^{2}\right) \lambda_{1}\left(P_{n}^{\mathrm{reg}}\right),
$$

with equality only for the regular n-polygon.

\section{DISCUSSION}

As was mentioned in the introduction, our numerical studies point to the fact that the upper bound given by (5-5) remains bounded as $n$ goes to infinity, suggesting a possible upper bound for more general domains, which is clear from Conjecture 5.4. Based on further numerical results we conjecture that it will in fact hold for simply connected domains.

Conjecture 6.1. For any planar simply connected domain $\Omega$ we have

$$
\lambda_{1}(\Omega) \leq \frac{\pi j_{01}^{2}}{A}+\frac{\pi^{2}}{4} \frac{L^{2}-4 \pi A}{A^{2}} .
$$


In this case, equality holds if $\Omega$ is either a disk or, asymptotically, a rectangle with one side length going to infinity. If true, the above conjecture would provide an improvement on the Pólya bound given by (1-3) in the case of simply connected domains, and also of the Payne and Weinberger bound (1-5). In fact, Conjecture 6.1 states that the sharp constant in bounds of the form of the Payne and Weinberger bound is actually what gives equality asymptotically for rectangles having the length of one side going to infinity.

To see that (6-1) always gives a better bound than $(1-5)$, it is sufficient to notice that this is equivalent to having

$$
\begin{aligned}
& \frac{\pi j_{01}^{2}}{A}+\frac{\pi^{2}}{4} \frac{L^{2}-4 \pi A}{A^{2}} \\
& \quad \leq \frac{\pi j_{0,1}^{2}}{A}\left[1+\left(\frac{1}{J_{1}^{2}\left(j_{0,1}\right)}-1\right)\left(\frac{L^{2}}{4 \pi A}-1\right)\right]
\end{aligned}
$$

which in turn is equivalent to

$$
1 \leq \frac{j_{01}^{2}}{\pi^{2}}\left(\frac{1}{J_{1}^{2}\left(j_{01}\right)}-1\right) \approx 1.58817
$$

If true, the above conjecture would also provide an improvement on the Pólya bound given by (1-3) in the case of simply connected domains.

We also remark that the conjecture is false if doubly connected domains are allowed. To see this, we considered the domain $H_{1}=D_{1} \backslash D_{2}$, where $D_{1}$ and $D_{2}$ are concentric balls with respective radii 4 and 1.3 , which are shown in Figure 9. The value of $\lambda_{1}\left(H_{1}\right)$ together with the corresponding values given by $(6-1)$ and Pólya's bound are shown in Table 5.
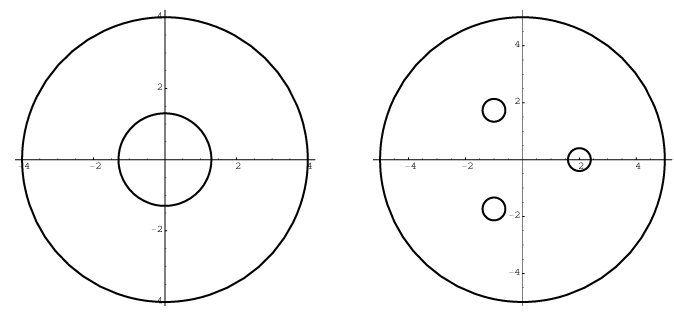

FIGURE 9. The domains $H_{1}$ and $H_{3}$.

\begin{tabular}{|c|c|c|}
\hline & $H_{1}$ & $H_{3}$ \\
\hline$\lambda_{1}$ & 1.31398304 & 0.68710829 \\
\hline Conj. 6.1 & 1.06829136 & 0.46436210 \\
\hline Pólya & 1.35385519 & 0.63101864 \\
\hline
\end{tabular}

TABLE 5. Values for $\lambda_{1},(6-1)$, and Pólya's bound for domains $H_{1}$ and $H_{3}$.
We also considered a domain $H_{3}$ with three holes, which is the ball with center at the origin and radius 5 , with three circular holes of radius 0.4 with centers at the points $(-1, \sqrt{3}),(-1,-\sqrt{3})$, and $(2,0)$, for which the value provided by Pólya's expression no longer gives an upper bound, but we were unable to find a similar example with only two holes. From the numerical point of view, as was proven in [Chen et al. 05], the method of fundamental solutions needs a special treatment when one is dealing with multiply connected domains, since then there might exist spurious eigenvalues. However, this problem never occurs in calculating the first eigenvalue.

We proceeded as in the case of the triangles to show that these domains provide counterexamples to Conjecture 6.1 and the upper bound (1-3). The results obtained for the domains $H_{1}$ and $H_{3}$ are shown in Table 6 .

\begin{tabular}{|c|c|c|}
\hline & $H_{1}$ & $H_{3}$ \\
\hline $\sup _{x \in \partial \Omega} \epsilon(x)$ & $4.91215 \times 10^{-9}$ & $2.03185 \times 10^{-12}$ \\
\hline$\|\tilde{u}\|_{L^{2}(\Omega)}$ & 5.30986 & 5.02033 \\
\hline
\end{tabular}

TABLE 6. Results for the approximations of the eigenfunctions of $H_{1}$ and $H_{3}$.

Applying the bounds (3-7) we obtain

$$
1.31398303186<\lambda_{1}\left(H_{1}\right)<1.31398304814
$$

and

$$
0.68710829922007<\lambda_{1}\left(H_{3}\right)<0.68710829922495,
$$

showing that these domains provide the desired counterexamples.

\section{ACKNOWLEDGMENTS}

We would like to thank Mark Ashbaugh for the many suggestions that helped improve the manuscript, and also for having pointed out many relevant references. We would also like to thank Carlos Alves for many helpful conversations. Our work was partially supported by FCT, Portugal, through programs POCTI/MAT/60863/2004 and POCTI/POCI2010.

\section{REFERENCES}

[Alves 00] C. J. S. Alves. "Density Results for the Helmholtz Equation and the Method of Fundamental Solutions." In Advances in Computational Engineering 8 Sciences, Vol. I, edited by S. N. Atluri and F. W. Brust, pp. 45-50. Forsyth, GA: Tech Sc. Press, 2000.

[Alves and Antunes 05] C. J. S. Alves and P. R. S. Antunes. "The Method of Fundamental Solutions Applied to the Calculation of Eigenfrequencies and Eigenmodes of 2D Simply Connected Shapes." Computers, Materials \& Continua 2 (2005), 251-266. 
[Brooks and Waksman 87] R. Brooks and P. Waksman. "The First Eigenvalue of a Scalene Triangle." Proc. Amer. Math. Soc. 100 (1987), 175-182.

[Chen et al. 05] J. T. Chen, I. L. Chen, and Y. T. Lee. "Eigensolutions of Multiply Connected Membranes Using the Method of Fundamental Solutions." Engineering Analysis with Boundary Elements 29:2 (2005), 166-174.

[Fox et al. 67] L. Fox, P. Henrici, and C. B. Moler. "Approximations and Bounds for Eigenvalues of Elliptic Operators." SIAM J. Numer. Anal. 4 (1967), 89-102.

[Freitas 06] P. Freitas. "Upper and Lower Bounds for the First Dirichlet Eigenvalue of a Triangle." Proc. Amer. Math. Soc. 134 (2006), 2083-2089.

[Hersch 66] J. Hersch. "Contraintes rectilignes parallèles et valeurs propres de membranes vibrantes." Z. Angew. Math. Phys. 17 (1966), 457-460.

[Hooker and Protter 60/61] W. Hooker and M. H. Protter. "Bounds for the First Eigenvalue of a Rhombic Membrane." J. Math. and Phys. 39 (1960/1961), 18-34.

[Kuttler and Sigillito 85] J. R. Kuttler and V. G. Sigillito. Estimating Eigenvalues with A Posteriori/A Priori Inequalities, Pitman Research Notes in Mathematics, 135. Boston: Pitman, 1985.

[Makai 62] E. Makai. "On the Principal Frequency of a Membrane and the Torsional Rigidity of a Beam." In Studies in Mathematical Analysis and Related Topics: Essays in Honor of George Pólya, pp. 227-231. Stanford: Stanford Univ. Press, 1962.
[Makai 59] E. Makai. Bounds for the Principal Frequency of a Membrane and the Torsional Rigidity of a Beam." Acta Sci. Math. Szeged 20 (1959), 33-35.

[Moler 69] C. B. Moler. "Accurate Bounds for the Eigenvalues of the Laplacian and Applications to Rhombical Domains." Stanford Technical Report CS 69-121, Stanford University, February 1969.

[Moler and Payne 68] C. B. Moler and L. E. Payne. "Bounds for Eigenvalues and Eigenfunctions of Symmetric Operators." SIAM J. Numer. Anal. 5 (1968), 64-70.

[Osserman 77] R. Osserman. "A Note on Hayman's Theorem on the Bass Note of a Drum." Comment. Math. Helvetici 52 (1977), 545-555.

[Payne and Weinberger 61] L. E. Payne and H. F. Weinberger. "Some Isoperimetric Inequalities for Membrane Frequencies and Torsional Rigidity." J. Math. Anal. Appl. 2 (1961), 210-216.

[Pólya 60] G. Pólya. Two More Inequalities between Physical and Geometrical Quantities." J. Indian Math. Soc. (N.S.) 24 (1960), 413-419.

[Pólya and Szegő 51] G. Pólya and G. Szegö. Isoperimetric Inequalities in Mathematical Physics, Annals of Mathematical Studies, 27. Princeton: Princeton University Press, 1951.

[Protter 81] M. H. Protter. "A Lower Bound for the Fundamental Frequency of a Convex Region." Proc. Amer. Math. Soc. 81 (1981), 65-70.

[Stadter 66] J. T. Stadter. "Bounds to Eigenvalues of Rhombical Membranes." SIAM J. Appl. Math. 14 (1966), 324341.

Pedro Antunes, CEMAT and Department of Mathematics, Instituto Superior Técnico, Av. Rovisco Pais, 1049-001 Lisboa, Portugal. (pant@math.ist.utl.pt)

Pedro Freitas, Department of Mathematics, Faculdade de Motricidade Humana (TU Lisbon) and Mathematical Physics Group of the University of Lisbon, Complexo Interdisciplinar, Av. Prof. Gama Pinto 2, P-1649-003 Lisboa, Portugal (freitas@cii.fc.ul.pt)

Received November 17, 2004; accepted in revised form December 7, 2005. 